\title{
Effect of telmisartan on hypertensive dementia patients: an observational study
}

\author{
Umashanker Prasad Keshri, Ravi Ranjan*, Risi Tuhin Guria, \\ Paramveer Kumar, Kumar Rajnish, Manju Gari
}

Department of Pharmacology, Rajendra Institute of Medical Sciences, Ranchi, Jharkhand, India

Received: 27 April 2020

Revised: 16 June 2020

Accepted: 17 June 2020

*Correspondence:

Dr. Ravi Ranjan,

Email: ravi4ranjan@gmail.com

Copyright: () the author(s), publisher and licensee Medip Academy. This is an open-access article distributed under the terms of the Creative Commons Attribution Non-Commercial License, which permits unrestricted non-commercial use, distribution, and reproduction in any medium, provided the original work is properly cited.

\begin{abstract}
Background: This was a prospective observational study done to observe the effects of telmisartan on cognitive function in hypertensive dementia patients.

Methods: The study included new diagnosed hypertensive and normotensive dementia patients. Patients comprised of four groups; hypertensive dementia patients taking telmisartan (group 1), hypertensive dementia patients taking donepezil and telmisartan (group 2), normotensive dementia patients taking donepezil (group 3) and normotensive dementia patients taking drugs which does not affect memory (group 4). Cognitive function of four groups were compared to each other at 4,8 and 12 weeks.

Results: In group 1, 2 and 3 mini-mental state examination (MMSE) scores has increased by 1.69, 16.81, and 13.28 percent and in group 4 there is decrease in MMSE score by 6.03 percent in 12 weeks.

Conclusions: In this study we can conclude that telmisartan has dementia preventing propensity which is better than placebo but not as good as donepezil.
\end{abstract}

Keywords: Telmisartan, Hypertensive dementia, Mini-mental state examination score

\section{INTRODUCTION}

Dementia is a wide range of symptoms associated with a decline in memory or other thinking skills severe enough to reduce a person's ability to perform everyday activities. It is marked by progressive cognitive impairment in clear consciousness. The cognitive function declines from previous level of functioning. It involves multiple cognitive domains including memory thus causing a significant impairment in social or occupational functioning compared to mild cognitive impairment (MCI) which is similar but does not cause significant impairment in functioning. ${ }^{1}$ Dementia is a complex and multifactorial disease.
Risk factors of dementia have been classified into modifiable and non-modifiable risk factors. Modifiable risk factors are vascular disease, hypertension, diabetes, dyslipidemia, depression, alcohol, smoking, obesity etc. Non-modifiable risk factors are age, family history, Apo E4 allele, female sex, head trauma, Down's syndrome, mutation of 1,4 and 21chromosomes. Prevalence of all types of dementia increases with increasing age. In some other recent study, it was found that users of ARBs had superior memory performance over time compared with other antihypertensive drug users.

Hypertension is one of the important risk factors for dementia. ${ }^{2}$ Telmisartan, an angiotensin-receptor blocker (ARB) is commonly used for treatment of 
hypertension. Two well characterized receptors are angiotensin type 1 receptor (AT1 receptor) and type 2 receptor (AT2 receptor). $\mathrm{AT}_{1}$ inhibition reduces cardiac and vascular oxidative stress, inflammation and remodeling, thus improving endothelial dysfunction, increased angiotensin II activity on $\mathrm{AT}_{2}$ receptors, leading to vasodilation and natriuresis through bradykinin, nitric oxide, prostaglandin and cyclic guanosine monophosphate (GMP) pathways, generally showing an opposite effect to the action of $\mathrm{AT}_{1}$ receptors. Recently renin-angiotensin-aldosterone system (RAAS) has been found to have an important role in cognitive function.

ARB blocks $\mathrm{AT}_{1}$ receptors also resulting in $\mathrm{AT}_{2}$ activation and contributes to some protective effects of ARBs. In the brain, the activation of $\mathrm{AT}_{2}$ reduces inflammation, superoxide production and axon degeneration, promotes neuronal cell differentiation and leads to activation of the repair systems. Several animal studies suggest the benefits of ARBs on cognitive function. ${ }^{3}$

Clinical study had been done on effect of telmisartan and ACE inhibitors on different aspect of dementia. The result is not very conclusive.

\section{METHODS}

It was an observational, prospective, open-label, noninterventional study. This was conducted in the Department of Pharmacology and Therapeutics, Rajendra Institute of Medical Sciences (RIMS), Ranchi amongst the patients attending outpatient and indoor Departments of Medicine and Psychiatry of Rajendra Institute of Medical Sciences (RIMS), Ranchi; Central Institute of Psychiatry, Kanke, Ranchi and Ranchi Institute of Neuro Psychiatry and Allied Sciences (RINPAS), Kanke, Ranchi.

\section{Ethical considerations}

Prior approval from Institutional ethics committee was taken before study and consent was taken from all patients on separate consent form for each patient.

\section{Study duration}

The study was conducted from March 2018 till October 2019. Every participant was followed up for 12 weeks in totality.

\section{Inclusion criteria}

Inclusion criteria were newly diagnosed hypertensive (systolic blood pressure $\geq 140 \mathrm{mmHg}$ and/or a diastolic blood pressure $\geq 90 \mathrm{mmHg}$ ) and normotensive (systolic blood pressure $<140 \mathrm{mmHg}$ and/or a diastolic blood pressure $<90 \mathrm{mmHg}$ ) dementia patients (MMSE score range 11-25) of age 55 years and above of both sexes suffering from mild to moderate dementia.

\section{Exclusion criteria}

Exclusion criteria were patients who were suffering from critical diseases and required emergency management or with co-morbidities other than hypertension (cardiac, renal, hepatic disorders and other psychiatric illness), HIV infected patients, patients taking drugs which affects memory like antidepressants, antipsychotic, betablockers, antihistaminics, benzodiazepines, antiepileptics, narcotics, antiparkinsonian drugs; patients with hypersensitivity to telmisartan and/or donepezil.

The patients were divided into four groups comprising thirty patients in each group; first two groups were hypertensive patients and last two groups were non hypertensive patients. The groups are as follows.

Group 1: Dementia patients taking telmisartan (dose 40 mg per day).

Group 2: Dementia patients taking telmisartan and donepezil (dose of telmisartan $40 \mathrm{mg}$ per day and donepezil $5 \mathrm{mg}$ per day).

Group 3: Dementia patients taking donepezil (dose $5 \mathrm{mg}$ per day).

Group 4: Dementia patients not taking drugs which affects memory but taking some other drugs, this group will be known as placebo group.

In this group those patients were included who came to OPD for various problems and found suffering from dementia and not had willingness for the treatment of this condition.

The mini-mental state examination (MMSE) scores were used as parameter for memory test at baseline and at intervals of $4^{\text {th }}, 8^{\text {th }}$ and $12^{\text {th }}$ weeks. It is a 30 points questionnaire used extensively in clinical and research settings to measure cognitive impairment and also as a screening test for dementia. It is also used to estimate the severity and progression of cognitive impairment and to follow the course of cognitive changes in an individual over time. Increase in MMSE score is indicative of cognitive improvement. ${ }^{4,5}$

Blood pressure was also measured with the help of mercury sphygmomanometer at baseline and same follow-up intervals of all patients.

\section{Statistical analysis}

Statistical analysis was done using the software statistical package for the social sciences (SPSS) version 17. Anova and Tukey's post hoc test was done. 


\section{RESULTS}

Demographic data of patients is presented group wise in (Table 1). Overall, there were total 45 females and 75 males in all four groups and low education level (illiterate and primary level of education) was found in $76 \%$ of cases. In group 1, 2 and 3 MMSE score has increased by $1.69,16.81$, and 13.28 percent and in group 4 there is decrease in MMSE score by 6.03 percent in 12 weeks.

Table 1: Demographic data of the patients.

\begin{tabular}{|c|c|c|c|c|c|}
\hline Characteristics & Group 1 & Group 2 & Group 3 & Group 4 & $P$ value \\
\hline Age in years $($ mean $\pm S D)$ & $65.80 \pm 7.09$ & $68.73 \pm 6.62$ & $66.60 \pm 6.63$ & $64.63 \pm 6.60$ & 0.116 \\
\hline \multicolumn{5}{|l|}{ Gender } & \multirow{3}{*}{0.399} \\
\hline Male & 10 & 11 & 15 & 9 & \\
\hline Female & 20 & 19 & 15 & 21 & \\
\hline \multicolumn{5}{|l|}{ Education level } & \multirow{6}{*}{0.523} \\
\hline Illiterate & 8 & 8 & 8 & 8 & \\
\hline Primary & 18 & 11 & 15 & 16 & \\
\hline Higher secondary & 4 & 6 & 3 & 2 & \\
\hline Graduate & & 4 & 4 & 4 & \\
\hline Postgraduate & & 1 & & & \\
\hline \multicolumn{5}{|l|}{ Economic status } & \multirow{4}{*}{0.212} \\
\hline Lower & 19 & 13 & 20 & 13 & \\
\hline Middle & 11 & 17 & 10 & 16 & \\
\hline Higher & & & & 1 & \\
\hline \multicolumn{5}{|l|}{ Habitat } & \multirow{3}{*}{0.825} \\
\hline Rural & 20 & 21 & 21 & 18 & \\
\hline Urban & 10 & 9 & 9 & 12 & \\
\hline
\end{tabular}

Table 2: Mean mini-mental state examination scores group-wise at 4,8 and 12 weeks.

\begin{tabular}{|llllll|}
\hline \multirow{2}{*}{ MMSE } & Group 1 & Group 2 & Group 3 & Group 4 & P value \\
\cline { 2 - 6 } Baseline & $19.50 \pm 4.14$ & Mean \pm SD & Mean \pm SD & Mean \pm SD & \\
\hline 4week & $19.63 \pm 4.24$ & $21.53 \pm 3.87$ & $19.13 \pm 4.38$ & $19.40 \pm 3.69$ & 0.966 \\
\hline 8week & $19.67 \pm 4.25$ & $21.83 \pm 3.77$ & $21.40 \pm 4.49$ & $19.50 \pm 3.84$ & 0.095 \\
\hline 12week & $19.83 \pm 4.14$ & $22.23 \pm 3.79$ & $21.67 \pm 4.51$ & $18.23 \pm 3.88$ & 0.001 \\
\hline
\end{tabular}

\section{DISCUSSION}

Our study found that there is no significant difference in mean MMSE score of telmisartan treated hypertensive dementia group (group 1) as compared to other three groups (2, 3 and 4$)$ at 4,8 and 12 th week (significance $0.11,0.31$ and 0.432 respectively).

Statistically the effect of telmisartan on hypertensive dementia patients is not significant in comparison to placebo in enhancing cognitive function but clinically it prevented decline in cognitive function. In the placebo group memory has declined by $6.03 \%$ (as evaluated by MMSE score) but there is no decline in memory in hypertensive dementia patients who were taking telmisartan. Instead in hypertensive dementia patients taking telmisartan there was little gain of $1.69 \%$ in cognitive function.
Donepezil is an effective drug for dementia and it was found better when compared with telmisartan. When donepezil and telmisartan were used simultaneously telmisartan showed the potentiating effect in dementia prevention.

Several mechanisms have been proposed to account for the apparent protective effect of ARBs, including their role in remodeling of cerebral microvasculature , inducing neural differentiation and DNA repair , reversing oxidative stress and inflammation, and preventing ischemic brain injury. ${ }^{6,7,8}$ It is supportive of our study because hypertensive dementia group (group 1) taking telmisartan (an ARB) had no decline in mean cognitive score when compared to the normotensive dementia patients taking no telmisartan or no donepezil (group 4). 
Telmisartan along with donepezil (group 2) and donepezil alone (group 3) both enhanced cognitive effects compared to group 1 and group 4, group 2 having significant effect because of additional effect of telmisartan which has different mechanisms of action which results in cognitive improvement.

Donepezil is a standard dementia drug approved by FDA. ${ }^{9}$ It has role of reference drug in this study. ${ }^{10}$ It acts by inhibiting acetylcholinesterase enzyme increasing the amount of acetylcholine in brain which increase cognition and memory. Accordingly, MMSE score in group-3 are significantly better at $8^{\text {th }}$ and $12^{\text {th }}$ weeks in comparison to group 4 (p values 0.029 and 0.008 respectively).

Cutuli et al showed that donepezil pre-treatment had beneficial effects on behavioral deficits induced by cholinergic depletion in rats.

Cholinergic depletion increased hippocampal and neocortical caspase-3 activity and impaired working memory, spatial discrimination, social interactions and behavior in rats. ${ }^{11}$

In a study patient with probable Alzheimer's disease (AD) and essential hypertension were randomly assigned to the telmisartan group or the amlodipine group (daily) for 6 months. The groups had a similar significant reduction in systolic and diastolic blood pressure after treatment. The telmisartan group did not show any changes in cognitive function test scores, while the amlodipine group showed significant decline on the AD assessment scale cognitive subscale. ${ }^{12}$ This indicates that telmisartan may have additional benefits and be useful for the treatment of elderly hypertensive patients with dementia.

One brain autopsy series study showed that ARB use was associated with significantly lower AD pathology, while no alteration of vascular pathology was observed when compared to other or no antihypertensive medication users. ${ }^{13}$

In animal study it has been found that low dose of telmisartan has a protective effect on the cognitive decline via up-regulation of brain-derived neurotrophic factor $(\mathrm{BDNF})$ and its receptor tropomyosinrelated kinase B (BDNF/TrkB) in the hippocampus of stroke-prone spontaneously hypertensive rats( SHRSPs) without hypotensive effect. ${ }^{14}$

Telmisartan has a unique binding mode to the AT1 receptor due to its distal benzimidazole portion. This portion could explain the highest lipophilicity, the greatest volume distribution and the strongest binding affinity of telmisartan to AT1 receptor. Furthermore, telmisartan was found to firmly bind to the AT1 receptor through the unique "delta lock" structure. A study suggests that due to its "delta lock" structure, telmisartan may be superior to other ARBs in halting cardiovascular disease in patients with hypertension. Cardiovascular disorders are one of the important risk factors of dementia and its progression. ${ }^{15}$

In the study on cognition and prognosis in the elderly (SCOPE) to assess whether candesartan-based ARB antihypertensive treatment in elderly patients with mildly to moderately elevated blood confers a reduction in cardiovascular events, cognitive decline and dementia, it was found that cognitive function was well maintained in both treatment groups in the presence of substantial blood pressure reductions. ${ }^{16}$ It correlates with our study.

Users of ARBs or ACEIs (blood brain barrier crossing medications) showed better memory performance over time than all other groups, including normotensives, and had less white mater hyper intensity volume over time than users of non- blood brain barrier crossing medications. ${ }^{17}$

In a recent longitudinal analysis of cerebrospinal fluid biomarker data from Alzheimer's disease neuroimaging initiative, attenuation of cerebral amyloid retention and progression to dementia among older adults taking ARBs were reported. $^{18}$

ACEIs reduce the amount of free angiotensin II and decrease damaging AT1 receptor activity, they also reduce beneficial AT2 receptor activity. ARBs block AT1 receptors and not the production of angiotensin II, they promote AT2 receptor activity and are "angiotensinconverting enzyme-sparing," allowing ACE to continue its suggested A $\beta$-degrading function, unlike ACEIs. ${ }^{19,20}$

Researchers in a nested case-control study of adults aged 60 years or older, who had either Alzheimer's disease, vascular dementia, or unspecified/other dementia, reported that patients taking ARBs and ACEIs had 53\% and 24\% lower risks of Alzheimer's disease, respectively, than those taking other antihypertensive medications. ${ }^{21}$

However, large clinical intervention trials such as ON TARGET (ongoing telmisartan alone and in combination with ramipril global endpoint trial) and TRANSCEND (telmisartan randomized assessment study in ACE intolerant subjects with cardiovascular disease) have not found significant differences in the incidence of dementia (which was one of its secondary outcome) between groups taking ARBs, ACEIs, or placebo. ${ }^{22}$

The PROGRESS (perindopril protection against recurrent stroke) study did not find a clear effect of hypertensive treatment on dementia, but reduced the risk of cognitive decline by approximately $20 \%$ and reduced the risk of "cognitive decline with recurrent stroke" by approximately $50 \% .^{23}$

Recently, telmisartan, an ARB, was found to act as a partial agonist of peroxisome proliferator-activated 
receptor-gamma (PPAR-gamma) ${ }^{24}$ PPAR-gamma influences the gene expression involved in carbohydrate metabolism, and pioglitazone and rosiglitazone, ligands for PPAR-gamma, improve insulin resistance in diabetic patients. Activators of PPAR-gamma exert antiinflammatory, anti-oxidative and anti-proliferative effects on vascular wall cells, thus decreasing the risks for atherosclerosis. It has also been hypothesized here that due to its unique PPAR-gamma-modulating activity, telmisartan will become a promising cardiometabolic sartan, that targets both diabetes and CVD in hypertensive patients. ${ }^{25}$

Recently, a Phase-1trial is going on to determine if telmisartan affects cognition and biomarkers of Alzheimer's in African Americans who is hypertensive and at risk of dementia and their parent or biological family member with Alzheimer's disease. Another trial of losartan (phase-2) to test the efficacy of reducing pathology in Alzheimer's Disease through angiotensin targeting (RADAR) is going on in mild to moderate Alzheimer's disease cases. ${ }^{26}$ These clinical studies will be helpful in revealing the role of telmisartan and ACE inhibitors in dementia prevention.

\section{CONCLUSION}

In this study we can conclude that telmisartan has dementia preventing propensity which is better than placebo but not as good as donepezil. The effect on cognition was better in the hypertensive dementia patients who were taking donepezil and telmisartan than the normotensive dementia patients who were taking only donepezil. This also shows potentiating effect of telmisartan on cognition.

There is need of clinical study with more specific tools and sensitive methods in a big sample size for longer period to establish telmisartan as dementia preventing drug in hypertensive dementia patients.

\section{ACKNOWLEDGEMENTS}

We are thankful to Dr Devesh Kumar of Department of Preventive and Social Medicine, RIMS, Ranchi for his valuable guidance.

\section{Funding: No funding sources} Conflict of interest: None declared

Ethical approval: The study was approved by the Institutional Ethics Committee, RIMS, Ranchi

\section{REFERENCES}

1. Richards SS, Sweat RA. Dementia. In Saddock NJ, Saddock VA, Ruiz P, editors. Kaplan and Sadock's Comprehensive Textbook of Psychiatry. 9th ed. Philadelphia: Lippincot Williamams and Willkins; 2009: 1167.
2. Kennelly SP, Lawlor BA, Kenny RA. Blood pressure and dementia: a comprehensive review. Ther Adv Neurol Disord. 2009;2(4):241-60.

3. Mogi M, Iwanami J, Horiuchi M. Roles of Brain Angiotensin II in Cognitive Function and Dementia. Int J Hypertens. 2012;169649.

4. Tombaugh TN, McIntyre NJ. The Mini-Mental State Examination: A Comprehensive Review. J Am Geriatrics Society. 1992;40:922-35.

5. Folstein M, Folstein S, Hugh MP. 'Mini-Mental State': a practical method for grading the cognitive state of patients for the clinician. J Psychiatr Res. 1975;2:89-98.

6. Wright JW, Harding JW. The brain RAS and Alzheimer's disease. Exp Neurol. 2010;223:326-33.

7. Villapol S, Saavedra JM. Neuroprotective effects of angiotensin receptor blockers. Am J Hypertens. 2015;28:289-99.

8. Mogi M, Horiuchi M. Effects of angiotensin II receptor blockers ondementia. Hypertens Res. 2009;32:738-40.

9. Cacabelos R. Donepezil in Alzheimer's disease: From conventional trials to pharmacogenetics. Neuropsychiatr Dis Treat. 2007;3:303-33.

10. Knowles J. Donepezil in Alzheimer's disease: an evidence-based review of its impact on clinical and economic outcomes. Core Evid. 2006;3:195-219.

11. Cutuli D, De BP, Caporali P, Tartaglione AM, Oddi D, Amato DFR, et al. Neuroprotective effects of donepezil against cholinergic depletion. Alzheimers Res Ther. 2013;24(5):50.

12. Kume K, Hanyu H, Sakurai H, Takada Y, Onuma T, Iwamoto T. Effects of telmisartan on cognition and regional cerebral blood flow in hypertensive patients with Alzheimer's disease. Geriatrics Gerontology Int. 2012;12(2):207-14.

13. Hajjar I, Brown L, Mack W, Chui H. Impact of angiotensin receptor blockers on Alzheimer's disease neuropathology in a large brain autopsy series. Arch Neurol. 2012;69:1632-8.

14. Kishi T, Hirooka Y, Sunagawa K. Telmisartan protects against cognitive decline via up-regulation of brain-derived neurotrophic factor/tropomyosinrelated kinase $\mathrm{B}$ in hippocampus of hypertensive rats. J Cardiology. 2012;60(6):489-94.

15. Ohno K, Amano Y, Kakuta H, Niimi T, Takakura S, Orita $\mathrm{M}$, et al. Unique 'delta lock' structure of telmisartan is involved in its strongest binding affinity to angiotensin II type 1 receptor. Biochem Biophys Res Commun. 2011;404(1):434-7.

16. Lithell H, Hansson L, Skoog I, Elmfeldt D, Hofman A, Olofsson B, et al. SCOPE Study Group. J Hypertension. 2003;21(5):875-86.

17. Ho JK, Nation DA. Memory is preserved in older adults taking AT1receptor blockers. Alz Res Therapy. 2017;9:33.

18. Nation DA, Ho J, Yew B. Older adults taking AT1receptor blockers exhibit reduced cerebral amyloid retention. J Alzheimers Dis. 2016;50:779-89. 
19. Kehoe PG, Miners S, Love S. Angiotensins in Alzheimer's disease - friendor foe. Trends Neurosci. 2009;32:619-28.

20. Oba R, Igarashi A, Kamata M, Nagata K, Takano S, Nakagawa $\mathrm{H}$. The $\mathrm{N}$-terminal active centre of human angiotensin-converting enzyme degrades Alzheimer amyloid $\beta$-peptide. Eur J Neurosci. 2005;21:733-40.

21. Davies NM, Kehoe PG, Shlomo BY, Martin RM. Associations of anti-hypertensive treatments with Alzheimer's disease, vascular dementia, and other dementias. J Alzheimer's Dis. 2011;26:699-708.

22. Anderson C, Teo K, Gao P, Arima H, Dans A, Unger $\mathrm{T}$, et al. Renin angiotensin system blockade and cognitive function in patients at high risk of cardiovascular disease: analysis of data from the on target and transcend studies. Lancet Neurol. 2011;10:43-53.

23. Tzourio C, Anderson C, Chapman N, Woodward M, Neal B, Mahon MS, et al. Effects of blood pressure lowering with perindopril and indapamide therapy on dementia and cognitive decline in patients with cerebrovasculardisease. Arch Intern Med. 2003;163:1069-75.

24. Benson SC, Pershadsingh HA, Ho CI, Chittiboyina A, Desai P, Pravenec M, et al. Identification of telmisartan as a unique angiotensin II receptor antagonist with selective PPAR gamma-modulating activity. Hypertension. 2004;43:993-1002.

25. Takagi, Hisato. Telmisartan as a metabolic sartan: The first meta-analysis of randomized controlled trials in metabolic syndrome. J Am Society Hypertension. 2013;7(3):229-35.

26. Kehoe PG, Blair PS, Howden B, Thomas DL. The Rationale and Design of the Reducing Pathology in Alzheimer's Disease through Angiotensin Targeting. Trial J Alzheimer's Dis. 2018;61(2):803-14.

Cite this article as: Keshri UP, Ranjan R, Guria RT, Kumar P, Rajnish K, Gari M. Effect of telmisartan on hypertensive dementia patients: an observational study. Int J Basic Clin Pharmacol 2020;9:1038-43. 